\title{
Optimizing Ballast Design of Wave Energy Converters Using Evolutionary Algorithms
}

\author{
Mitch Colby \\ Oregon State University \\ Corvallis, OR, 97331 \\ colbym@engr.orst.edu
}

\author{
Ehsan Nasroullahi \\ Oregon State University \\ Corvallis. OR, 97331 \\ nasroumo@engr.orst.edu
}

\author{
Kagan Tumer \\ Oregon State University \\ Corvallis, OR, 97331 \\ kagan.tumer@oregonstate.edu
}

\begin{abstract}
Wave energy converters promise to be a viable alternative to current electrical generation methods. However, these generators must become more efficient before wide-scale industrial use can become cost-effective. The efficiency of these devices is primarily dependent upon their geometry and ballast configuration which are both difficult to evaluate, due to slow computation time and high computation cost of current models. In this paper, we use evolutionary algorithms to optimize the ballast geometry of a wave energy generator using a two step process. First, we generate a function approximator (neural network) to predict wave energy converter power output with respect to key geometric design variables. This is a critical step as the computation time of using a full model (e.g., AQWA) to predict energy output prohibits the use of an evolutionary algorithm for design optimization. The function approximator reduced the computation time by over $99 \%$ while having an average error of only $3.5 \%$. The evolutionary algorithm then optimized the weight distribution of a wave energy generator, resulting in an $84 \%$ improvement in power output over a ballast-free wave energy converter.
\end{abstract}

\section{General Terms}

Algorithms; Applications

\section{Keywords}

Evolutionary algorithm; neural network; function approximation; optimization

\section{INTRODUCTION}

Due to the increase of population, demand for power, and the desire for economical stability, there is a high demand for renewable energy sources that are both environmentally friendly and economically viable. Wave energy is an energy source that satisfies both the political and economical demands, and can reduce the environmental impacts of power

Permission to make digital or hard copies of all or part of this work for personal or classroom use is granted without fee provided that copies are not made or distributed for profit or commercial advantage and that copies bear this notice and the full citation on the first page. To copy otherwise, to republish, to post on servers or to redistribute to lists, requires prior specific permission and/or a fee.

GECCO'11, July 12-16, 2011, Dublin, Ireland.

Copyright 2011 ACM 978-1-4503-0557-0/11/07 ...\$10.00. generation $[15,13]$. Wave energy belongs to a group of energy sources which are easy to access, but require a complex process to capture.

The issues of harnessing waves as viable sources of energy include the complicated nature of predicting the wave climate, as well as the difficulties associated with optimizing a wave energy converter (WEC) for a particular wave climate. System parameters including inertia, center of gravity position, system draft, and submerged volume all may affect the power output of a WEC. Mass parameters such as inertia and center of gravity position can be manipulated during operation by utilizing ballast, thus affecting system power output. Because of the complex simulations that they require, testing different design parameters is very time consuming, which limits the number of designs which may be considered.

Hydrodynamic simulators, such as Ansys AQWA, are capable of simulating different WEC configurations. However, these simulators are extremely time-consuming, due to the fact that the energy and momentum equations are solved thousands (or millions) of times during one simulation [1]. Thus, it is essential that the computation time of analyzing WECs is reduced, allowing for more designs to be analyzed during the optimization process.

In order reduce computational time, we developed a function approximation that maps the design configuration of a WEC to its power output (section 3.2). This reduction in computational time allows for a larger portion of the design space to be searched in a given amount of time. Given this function approximation, evolutionary algorithms can be utilized to find the ideal ballast configuration for a WEC in much less time than with traditional hydrodynamic analysis methods. Optimizing the ballast design for a WEC is an essential component to ensuring that the WEC is cost effective.

The geometry of a WEC has been defined and was developed by Columbia Power Technologies INC (Columbia Power). With this defined geometry, geometry parameters such as system draft or submerged volume are set, and cannot be changed. However, the mass parameters may be changed during operation in order to affect the power output. The power output of this particular WEC has been shown to be most sensitive to the inertia and center of gravity positions of each of its components. Installing ballast chambers in the WEC allows us to modify those values during the course of operation, which affects the power output of the device. However, there are countless possibilities for the ballast chamber configuration, so some type of search 
has been perform to find an optimal ballast chamber configuration which maximizes the WEC power output. An evolutionary search over ballast chamber configurations should yield a WEC design which is superior to a ballast-free model.

In this paper, we show how we developed a neural network function approximator that maps the mass parameters of each component of a WEC to the power output for that WEC (section 3.2). Next, a time-domain simulator was created with this function approximator, which predicted the annual energy output of a WEC given its ballast configuration. Finally an evolutionary algorithm was utilized to intelligently search through the set of potential ballast configurations, using the time-domain simulator to rank each design. This ultimately lead to the optimal ballast configuration within the WEC.

\section{BACKGROUND}

In order to optimize the ballast configurations of the wave energy converter to maximize energy output for a specific wave climate, we completed the following steps:

- Find the appropriate geometry for our WEC

- Simulate that geometry with different mass parameters using AQWA

- Use the simulated data to train the neural network function approximator

- Search through the different ballast cut configurations to find the optimal set of ballast cuts for a given wave climate.

In this section we discuss the WEC geometry and the hydrodynamic simulator, which is the foundation of our work. In section 3, we outline our methods and highlight our contributions to this research.

\subsection{Wave Energy Converter Geometry}

The specific geometry of the WEC for analysis was developed by Columbia Power. The WEC is a heaving and pitching design, which is based on the concept of the components resonating with the dominant wave frequency experienced in a given wave climate [11]. The WEC has three components as shown in the Figure 1. The components are the spar, the forward float, and the aft float.

\subsection{Simulation and Design}

Ansys AQWA is a powerful software package which simulates bodies floating in the ocean. Unlike traditional computational fluid dynamics packages, which utilize finite volume analysis, AQWA utilizes panel analysis to determine the hydrodynamic response of offshore and marine structures. Running a simulation in AQWA consists of generating a surface mesh, defining the mass properties of the body, and defining the wave climate [1]. The AQWA simulation outputs the hydrodynamic response of the defined body in the specified wave climate.

For the AQWA simulations, the y-moments of inertia $I_{y y}$ and the vertical center of mass positions $C G_{z}$ of each component of the WEC were chosen as analysis parameters, because the power output of the WEC has been shown to be most sensitive to the $I_{y y}$ and $C G_{z}$ parameters (mass parameters). The $\mathrm{y}$-moments of inertia were chosen because the

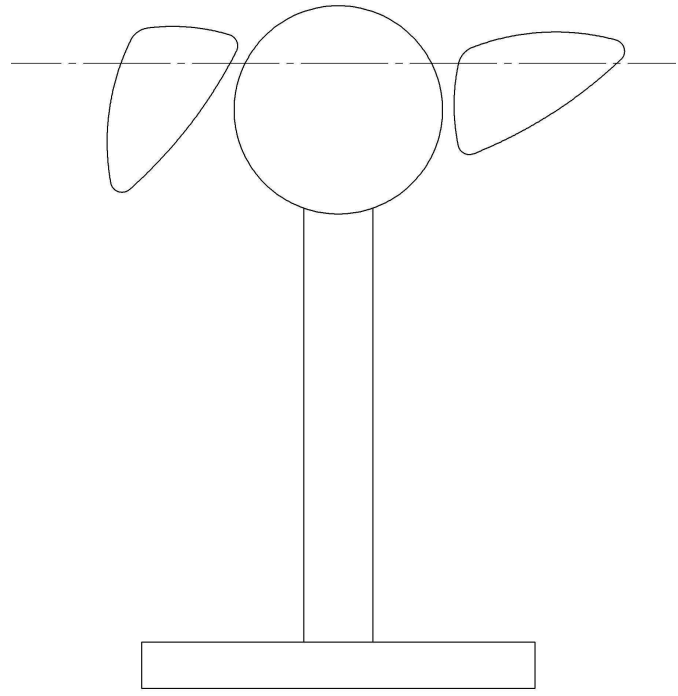

Figure 1: Columbia Power Manta WEC, which has three sections: the spar (the middle rode), aft float ( the top right float), and forward float (the top left float). The dashed line indicates the level-sea waterline

WEC design produces power when the components rotate about the y-axis, and the vertical center of mass positions were chosen because they affect hydrodynamic stability [17]. After defining the feasible bounds for each of the input parameters (based on feasible manufacturing processes), the input space was discretized into 400 points, and these points were simulated in AQWA to find the power output as a function $I_{y y}$ and $C G_{z}$ configuration, as well as the dominant wave frequency of the sea (assuming a specified wave climate).

\subsection{Related Work}

In this section we discuss related work which has been completed on neural network function approximation, evolutionary algorithms, and WEC optimization. Each specific topic has been studied in depth, but evolutionary algorithms and neural networks have not been widely utilized in WEC design.

Neural networks have been used in many applications, including control, system identification, function approximation, and nonlinear signal-processing $[3,14,8,10]$. Neural networks are excellent tools for finding input/output mappings from datasets, and have been shown to be capable of finding these mappings in highly nonlinear datasets [3]. A key advantage when using neural networks for function appoximation is that once trained, the neural network can be evaluated and make predictions almost instantaneously. This computational speed is a crucial component when performing a population based search such as an evolutionary algorithm.

Evolutionary algorithms have been successfully implemented to optimize design processes, including autonomously designing antennas, finding optimal gaits of quadruped robots, solving offset assignment problems for embedded processors, and developing distributed control policies in multi- 
component systems $[6,7,9,2]$. Evolutionary algorithms have proven to be useful in design in many very different domains. These designs indicate that evolutionary algorithms guiding design processes offer many advantages, including an autonomous process and the fact that no domain expert is necessary. Often, as design problems become more complex, a domain expert may impart more bias into the design process, greatly limiting the potential designs which may be considered. With an evolutionary algorithm, designs which a domain expert would never consider have the potential to be considered. Often, in these more complex domains, the evolutionary algorithms produce exceptional results when compared to traditional design methods $[2,4,18]$.

Wave energy converters are not a new technology, and designs from the 1970s such as Salter's Duck are still in use. Due to the complexities involved in wave energy converter design, coupled with the fact that hydrodynamic simulators are very time-consuming, most wave energy converter design processes are ad-hoc processes in which existing designs are slightly modified and tested in specific wave climates [11]. Due to the nature of these ad-hoc designs, formal algorithms for optimizing wave energy designs are rare. Often, "new" wave energy converter designs are simply modified models of existing wave energy converters [5]. While our research does concentrate on one WEC geometry, we are not implementing an ad-hoc algorithm. The use of an evolutionary algorithm in the design process removes the need for expert domain knowledge, because the evolutionary algorithm intelligently searches the space. Thus, there are two key differences between traditional WEC design processes and the process we implement. First, the design is guided by evolution, rather than a human expert. Secondly, the use of function approximators to replace simulations drastically increases the number of designs which may be considered, which makes the implementation of the evolutionary algorithm possible

\section{APPROACH}

In this section we present the experimental approach used in our research, which is based on the WEC geometry and AQWA simulations described in section 2. We develop a neural network function approximator and combine it with an evolutionary algorithm in order to optimize the WEC ballast configuration and maximize the power output for a specific wave climate.

First, the given geometry of the WEC was used to simulate power outputs in AQWA for multiple ballast configurations (section 3.1). Then, we use a neural network to map the mass parameters to power output of the WEC (section 3.2). Finally, we used an evolutionary algorithm to do an intelligent search through our search space and to optimize the ballast design (section 3.3).

\subsection{Ballast Chamber Geometries}

The floats utilized on the WEC are variations of Salter's Duck design, which has been shown to be capable of capturing $90 \%$ of a single wave's energy in ideal climates [12]. Although Salter's Duck float design is extremely efficient in an ideal climate, this ideal climate consists of constant frequency waves moving in one direction and is thus an unrealistic model for operation in the sea. In a true sea climate, the efficiency of the Duck design decreases significantly. It is impractical to change the WEC geometry during operation, but ballast can easily be altered in order to change mass parameters such as $I_{y y}$ and $C G_{z}$ values in order to alter the power output. It is essential that an optimal ballast configuration be found, in order to maximize energy extraction from a given sea state.

The entire volume of the wave energy converter is not available for use for ballast chambers. Practical constraints, such as placement of batteries, the generator, electrical equipment, and mass dampers limit the portion of the WEC which may be used for ballast chamber placement. Regions where ballast chambers should not be placed are shown as the unshaded regions of Figure 2. The main stem of the spar is available for use as a ballast chamber, as are the front and aft float bodies. The shaded regions in Figure 2 represent the areas available for use as ballast chambers. The objective is to split this volume into separate ballast chambers such that the annual power output of the WEC is optimized, given a set number of total ballast cuts. The reason that the number of ballast cuts is not a design variable is because if included as a design variable, the number of ballast chambers would tend to infinity. An infinite number of infinitesimal chambers which can instantly fill and empty would allow for near-instantaneous large changes of the inertia and center of gravity parameters, which is mathematically ideal to optimize the power output but is not practical from a manufacturing or cost standpoint.

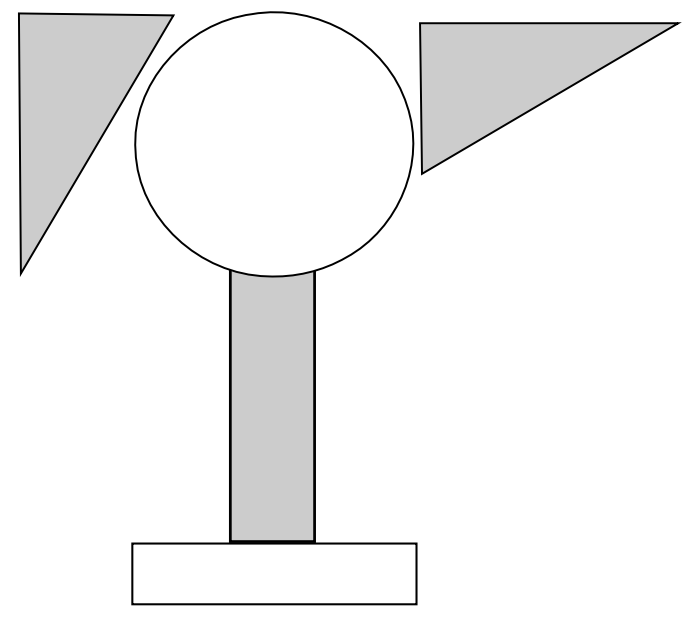

Figure 2: Shaded areas indicate ballast chamber locations

The volumes available for use as ballast are cut with planes to create the separate ballast chambers (Figure 3 ). The variables associated with these cuts are the position at which these cuts intercept the ballast volume. Given a set of cuts, a mapping from the mass of seawater (used for ballast) in each chamber to the overall component $I_{y y}$ and $C G_{z}$ values is found. Thus, for a given set of cuts, the inertia and center of mass of each component is simply a function of the ballast mass in each chamber created by the cuts.

Once a set of ballast cuts is determined, the desired mass of seawater in each ballast chamber is found as a function of the dominant wave frequency. The neural network function approximator described in section 3.2 provides the ideal $I_{y y}$ and $C G_{z}$ values for each dominant wave frequency. For a given set of ballast cuts, the masses of seawater in each ballast chamber which result in the WEC having the desired $I_{y y}$ and $C G_{z}$ values are found. So, for a set of ballast cuts, 


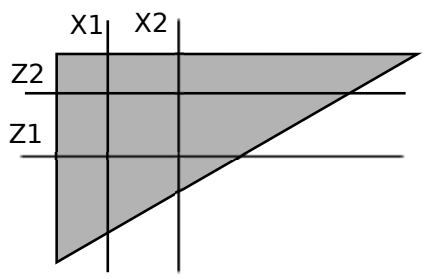

Float Ballast Cuts

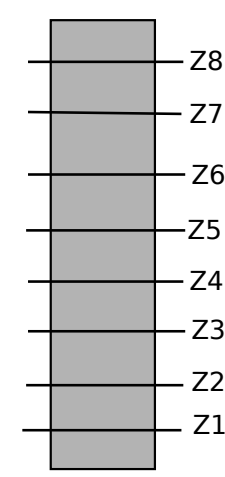

Spar Ballast Cuts

Figure 3: Cuts which create ballast chambers

the desired mass in each ballast chamber is known as a function of dominant wave frequency. These masses are used as set-points in the time domain simulation of the WEC.

\section{Wave Occurrence Prediction}

Wave energy converters must be optimized for a specific climate for several reasons. First, the WEC should resonate at the frequencies seen in the wave climate which it will operate in. Furthermore, predicting the future wave climate is necessary for properly controlling the ballast chambers of the WEC. It is impractical to constantly have ballast pumps activated during operation, so the WEC ballast should be set based on predictions of the future wave climate. Without an accurate prediction, the ballast settings will be suboptimal and power output would suffer. By designing the WEC for a specific wave climate, the ballast chamber geometry can be optimized for the waves expected in that climate, and the controller can set ballast weights based on accurate predictions.

The WEC is being optimized for use off of the Oregon coast. The wave climate in this region is defined by a wave occurrence table, which gives the total number of hours per year that each dominant wave frequency is present. From the wave occurrence table, a smooth sea and a rough sea are created. The smooth sea is the realistic wave climate based on the table, while the rough sea is a random permutation of the smooth sea. The random sea is a good test domain because it forces transient operation, which is what the ballast geometry choices have the greatest effect on. At steady state, different ballast configurations perform equally well, assuming that the ballast configurations allow for the ideal $I_{y y}$ and $C G_{z}$ values for each dominant wave frequency to be physically realizable. The smooth sea gives an idea of the expected annual power output of the WEC, while the random sea gives an idea of transient WEC performance and the relative efficacy of each ballast configuration which is analyzed.

\subsection{Neural-Network Function Approximation}

In order to create a neural network function approximator for the power output of the WEC, a training data set had to be created through simulations. In order to perform a large search, a function approximator is necessary to reduce computational time due to the simulations. AQWA is capable of finding the power output of the WEC, based upon the
$I_{y y}$ and $C G_{z}$ parameters of each component. However, the simulation process is extremely slow, taking up to 4 hours per simulation. In order to speed up the simulation process, a neural network was used as a function approximator for the power output portion of the AQWA simulator, for the particular set of geometries associated with characteristic of this particular WEC.

A neural network was used because the data set is highly nonlinear, and neural networks are excellent choices for finding patterns in nonlinear datasets $[14,8,16]$. Simpler function approximation techniques, including linear and polynomial regression, were unable to produce accurate mappings with our dataset.

The neural network function approximator maps the mass parameters $\left(I_{y y}\right.$ and $\left.C G_{z}\right)$ and dominant wave frequency to the WEC power output. Keep in mind that this approximator is a substitute for the part of AQWA that simulates power output based upon WEC mass parameters. In addition, it is only suited for the geometry of this particular WEC, so we are by no means replacing the entire AQWA simulator. This function approximation was only designed to reduce the simulation time for a specific WEC geometry and wave climate, which is over $99 \%$ reduction from original AQWA simulation run times.

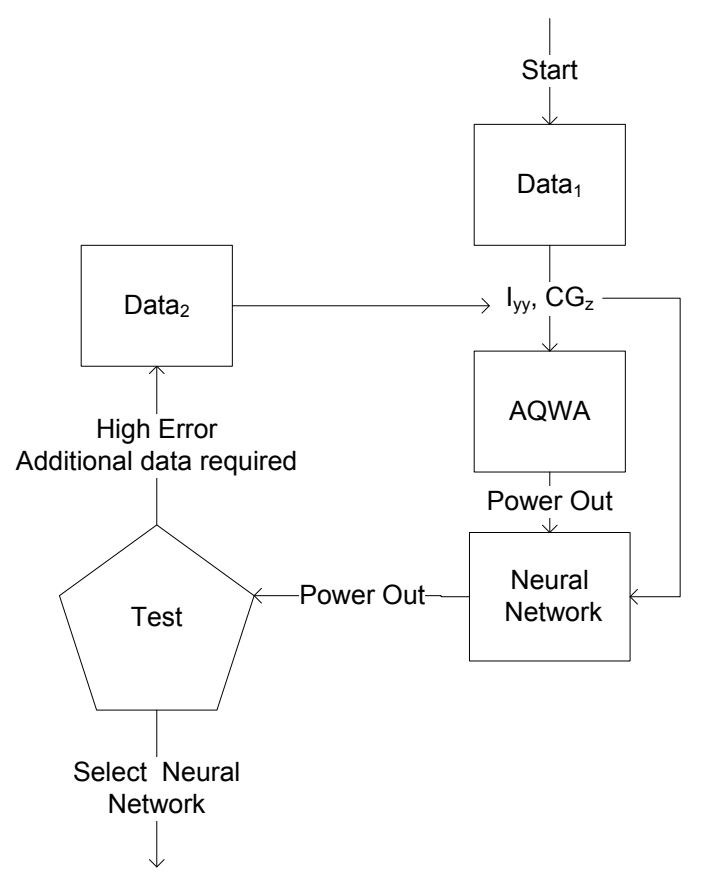

Figure 4: Neural Network Training: 250 data points (Data 1) were created uniformly across input space. These points were simulated in AQWA, giving the power output of each point. The neural network trained from this data was inadequate, so 150 new data points were simulated in AQWA (Data 2), in the region of the input space with highest uncertainty. The new dataset produced an accurate neural network function approximation

We used the AQWA simulator to generate a set of training data for the neural network. First, we looked at the manufacturability bounds on $I_{y y}$ and $C G_{z}$ so our training data 
would come from a realistic input space. The input space was discretized into 250 sets of data points $\left(I_{y y}\right.$ and $\left.C G_{z}\right)$, and each set was simulated in AQWA to get the resultant power output. We then needed to determine the appropriate number of hidden units to use in the neural network. The training data was fed into three separate neural networks with 5, 10, and 20 hidden units. The validation error on the 5 and 10 hidden unit neural networks was over $40 \%$, while the 20 hidden unit neural network had mean error of just $8 \%$ overall. There were certain regions of input values $\left(I_{y y}\right.$ and $C G_{z}$ ), which the error of the 20 hidden unit neural network rose to $20 \%$. Over these regions, we increased resolution and reduced the error by adding an additional 150 sets of data points (400 total sets of data points). Thus the neural network that resulted is a one hidden layer (20 hidden units) feed-forward network which was trained with backpropogation and a learning rate of 0.1 . 10-fold cross validation was used to validate network performance; the average error for was $1.5 \%$, and the maximum error was $6.12 \%$. This function approximator allows an exhaustive search through the entire input space, and was used to find the optimal $I_{y y}$ and $C G_{z}$ configurations for each dominant wave frequency.

\section{Time Domain Simulation}

For a given set of ballast cuts, the desired mass of seawater in each ballast chamber as a function of wave frequency is known. This information, along with the smooth and random sea models, was used to create a time-domain (TD) simulation of the WEC performance. Each ballast chamber has a water pump and a PID controller, and the WEC has a sensor which gives the dominant wave frequency in real time. Whenever there is a change in the dominant wave frequency, the set-points of the PID controllers are updated using the list of desired ballast masses. This process is continued until one year of operation is simulated, and the annual power output of the WEC is then calculated using the neural network base function approximator.

\section{Algorithm 1: Time domain simulator}

Start: time $=0$
While (time $<$ timeFinal)
1: Select Control Time
2: if new control loop started
$\quad \quad$ - Select period associated with control loop
$\quad \quad$ Find mass setpoints for ballast chambers
3: Find Mass Difference:
$\quad$ MD = MassSetpoint - MassCurrent
4: Find pump rates as a function of mass difference
5: Turn on pumps
6: Find instantaneous mass at time t
7: Calculating instantaneous power using our neural
$\quad$ network function approximator
8: Calculate energy produced by WEC
9: time++

\subsection{Evolutionary Algorithm Representation}

With the neural network function approximator and the time-domain simulator completed, an evolutionary algorithm was implemented by using these tools. Because the neural network saves so much computational time, a populationbased search such as an evolutionary algorithm is possible. An evolutionary algorithm is used to determine the ideal set of ballast cuts for the WEC. Each member of the population is a vector of cut positions. One population member a vector with 16 elements ( $2 \mathrm{x}$ - and $\mathrm{z}$-cut positions for the floats, and $8 \mathrm{z}$-cut positions for the spar). The mutation operator is two-fold. First, one to three indices are randomly selected (number of indices selected is randomly chosen, and the indices are randomly chosen). Second, the vector elements corresponding to the indices selected are mutated by a percentage determined from a Gaussian curve. The average mutation was $1 \%$, and the standard deviation of the normal curve was $0.33 \%$. The sign of the mutation was set to be positive or negative with equal probability. The rank of each population member is equivalent to the annual power produced (as found from the time-domain simulation) by a WEC with the ballast chamber geometry defined by that population member. An $\epsilon$-greedy policy is used to select population members at the end of each epoch, in order to avoid local minima which algorithms such as gradientdescent or hill-climbing are ill-equipped to handle $(\epsilon=0.05)$. For our experiments, the population size was 500 and the experiments were run for 1000 epochs over 25 statistical runs.

Algorithm 2: Evolutionary algorithm methodology for optimizing ballast cuts

1: Start from $\mathrm{n}$ random states $\left(K_{\text {current }}\right)$

2: Mutate each current state, resulting to population of size $2 n\left(K_{\text {suc }}\right)$

3: Rank each population member using TD simulation

4: Select $n$ states $\left(K_{n e w}\right)$ from the pool of $2 n$ states $\left(K_{\text {suc }}\right)$ using $\epsilon$-greedy, and set to current population

$$
K_{\text {current }}=K_{\text {new }}
$$

5: Go to step 2

\section{RESULTS}

In this paper, a series of three experiments were carried out in order to optimize the design of ballast chamber cuts. First, we trained a neural network to serve as a function approximator for AQWA for our particular WEC geometry, reducing computation time. Then we used the function approximator to test WEC geometries with and without ballast chambers, to observe the difference in performance. Next, using the power output from the neural network function approximator, we determined the optimal control loop time for the WEC. Finally, taking the neural network approximator as an evaluation function, and using the optimal control loop time, we ran an evolutionary algorithm to optimize the geometries of ballast cuts.

1. Trained a neural network 
2. With a control loop time of zero, ballast configuration from EA compared against a ballast-free WEC

3. Utilizing multiple control loops, ballast configurations from EA compared against ballast configurations from hill-climbing

\subsection{Neural Network Training}

The neural network function approximator was trained using data from the AQWA simulations (Table 1). With only 250 data points, the neural network had high error when used for prediction, regardless of the network topology utilized. 150 more data points were simulated in AQWA, in the areas of the input space with the highest error. With 400 data points, the neural network became very accurate, with $6.12 \%$ maximum error and $1.5 \%$ mean error, using 10 -fold cross-validation (error measured as percent difference between neural network prediction and AQWA output). Furthermore, $98 \%$ of the data set had error less than $5 \%$. For this particular WEC geometry, the trained neural network is accurate enough to replace AQWA for predicting power output as a function of the mass properties of the WEC.

\begin{tabular}{|c|l|l|l|l|}
\hline \multicolumn{5}{|c|}{ Neural Network Accuracy Table of Cross Validation Error } \\
\hline Data Points & $\begin{array}{l}\text { Hidden } \\
\text { Units }\end{array}$ & $\begin{array}{l}\text { Max } \\
\text { Error }\end{array}$ & $\begin{array}{l}\text { Mean } \\
\text { Error }\end{array}$ & $\begin{array}{l}\text { Data Set with } \\
<5 \% \text { Error }\end{array}$ \\
\hline 250 & 10 & $42.35 \%$ & $24.32 \%$ & $22.00 \%$ \\
\hline 250 & 15 & $36.53 \%$ & $18.26 \%$ & $36.00 \%$ \\
\hline 250 & 20 & $34.46 \%$ & $15.35 \%$ & $42.00 \%$ \\
\hline 400 & 10 & $14.22 \%$ & $8.62 \%$ & $89.00 \%$ \\
\hline 400 & 15 & $9.35 \%$ & $5.36 \%$ & $92.00 \%$ \\
\hline 400 & 20 & $6.12 \%$ & $1.50 \%$ & $98.00 \%$ \\
\hline
\end{tabular}

Table 1: Validation error for the neural network function approximator; the best network has a maximum error of $6.2 \%$ and mean error of $1.5 \%$. Reported errors are generated from 10 -fold cross validation

By creating a neural network which can be used to predict the WEC power output as a function of mass properties, we made it possible to perform an evolutionary algorithm on the ballast design. Because AQWA simulations are so time consuming, it would be infeasible to perform population-based searches such as evolutionary algorithms in the wave energy domain. However, a trained neural network can be evaluated essentially in real time, allowing for many more candidate solutions to be considered. Without the neural network function approximator, we could not have performed the rest of our experiments.

\subsection{Performance of Ballast-Free WEC}

Ballast chamber cut positions were evolved in order to maximize annual WEC energy capture, with a control loop time of zero (Figure 5). A population of cut positions was randomly initialized, and each population member was ranked based on how much annual energy it produced in the time domain simulation. At any time $t$ in the time domain simulation, the WEC sensed the dominant wave frequency and adjusted its ballasts accordingly. No control loop time was implemented, so the controller was not looking forward in time. For comparison, a WEC was tested which had no ballast chambers, but had the optimal $I_{y y}$ and $C G_{z}$ values associated with the average dominant wave frequency encountered throughout the year. This comparison gives an indication of the gains in power output that are associated with the ballast chambers.

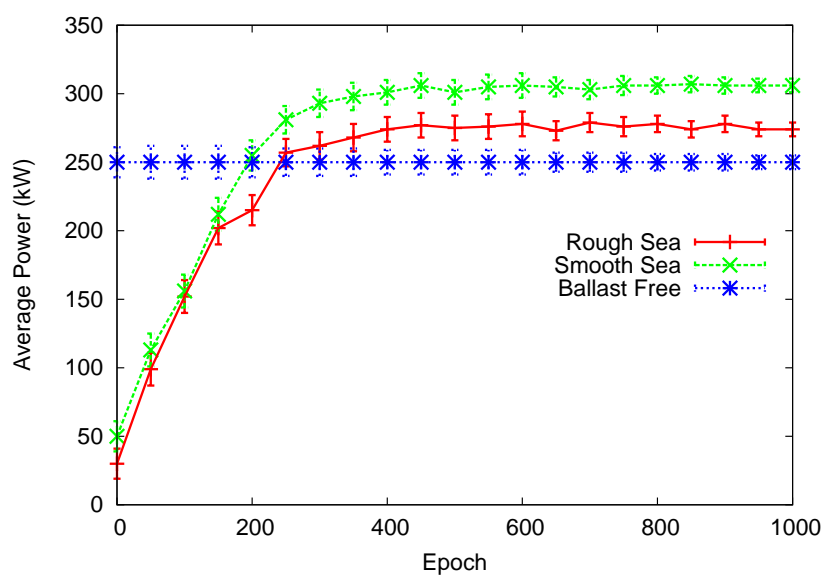

Figure 5: The evolutionary algorithm performance in two environments are compared against a ballastfree WEC which retains a static configuration during operation. The results shows that ballast chambers makes the average power increase up to $17 \%$ when compared to the ballast-free WEC. The smooth sea is a realistic sea climate based on the Oregon coast, and the rough sea is a random permutation of this sea climate. (Note: as the ballastfree model remains static throughout operation, it performs equally well in the rough and smooth seas, because they are permutations of each other)

A few interesting observations can be made from Figure 5. First, the WEC produces more power when operating in a smooth sea than when operating in a random sea. This is due to the fact that the WEC is producing the most power when operating in steady-state. In the random sea, the time spent in transient operation increased, reducing the power output. The random sea was selected as the primary testing domain, because the power output during transient operation is what ultimately differentiates one ballast design from another. The evolved WEC produced more power than the ballast-free WEC, regardless of whether it was operating in a smooth or random sea. This result indicates that an evolutionary algorithm can effectively guide the design of the WEC ballast chambers, increasing the total power output.

\subsection{Comparison of Control-Loop Times}

Next, ballast chamber cut positions were evolved while utilizing different control loop times. The WEC can alter its mass properties at any time by pumping water in and out of the ballast chambers. In the time domain simulator, the controller looks ahead some amount in time, and bases the ballast setpoints off the expected wave climate in that time interval. The maximum power produced (as found from evolution) as a function of the control loop time utilized is shown in Figure 6. Assuming that we knew the dominant wave frequency for $n$ time steps into the future, the dominant wave frequency was calculated as the mean of the future wave frequencies that will be experienced in the next $n$ time steps.

From Figure 6, we see that the choice of the control loop 


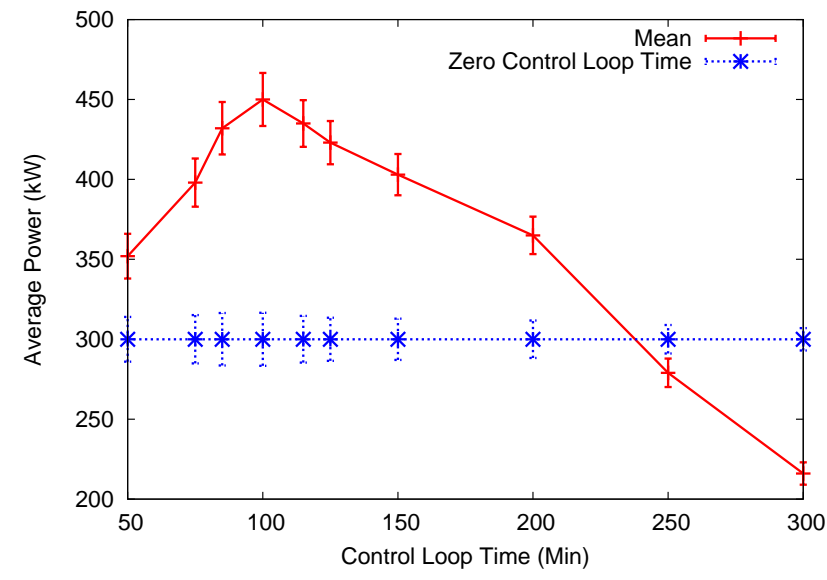

Figure 6: Average power output as a function of the control loop time. The constant line is the power output obtained when the control loop time was zero

time is critical to maximizing WEC power output. If the WEC has a short control loop time, it is constantly in transient operation, and power output suffers. If the WEC has too long of a control loop time, the prediction of the expected wave climate becomes inaccurate, also reducing the power output. The optimal control loop time is about 100 minutes in the specified sea climate.

Next, we compared the maximum WEC energy output found from an evolutionary algorithm to the energy output of a WEC produced through random-restart hill climbing (Figure 7). For the hill-climbing search, 500 points in the input space were randomly initialized. At each time step, all neighbor states of each point were generated by discretizing the cut-position space into 1 millimeter increments, and the point with the highest power output was chosen. We have found that evolutionary algorithms can be useful tools for ballast design, and that the control loop time is critical for optimal power output.

From Figure 7, we see that the ballast designs generated with evolution perform far better than the ballast designs generated with hill-climbing methods. With an optimized ballast design and control loop time, the WEC produces $84 \%$ more power than the ballast free model tested in Section 4.2.

\subsection{Computational cost of Neural Network}

Completing the evolutionary algorithm was only possible because of the speed of the neural network function approximator. Running the algorithm using AQWA would be prohibitively slow. At each time step in a time-domain simulation, the neural network is called to calculate the energy produced during that time step. The time domain simulation is embedded into the evolutionary algorithm, and runs for each population member at each time step. Evaluating the neural network once takes less than one second, while one AQWA simulation takes around four hours. A comparison of the time to run some experiments using the neural network or AQWA to calculate power is shown in Table 2.

In our experiments with evolving the ballast geometry, a population size of 500 run over 1000 epochs took around 6

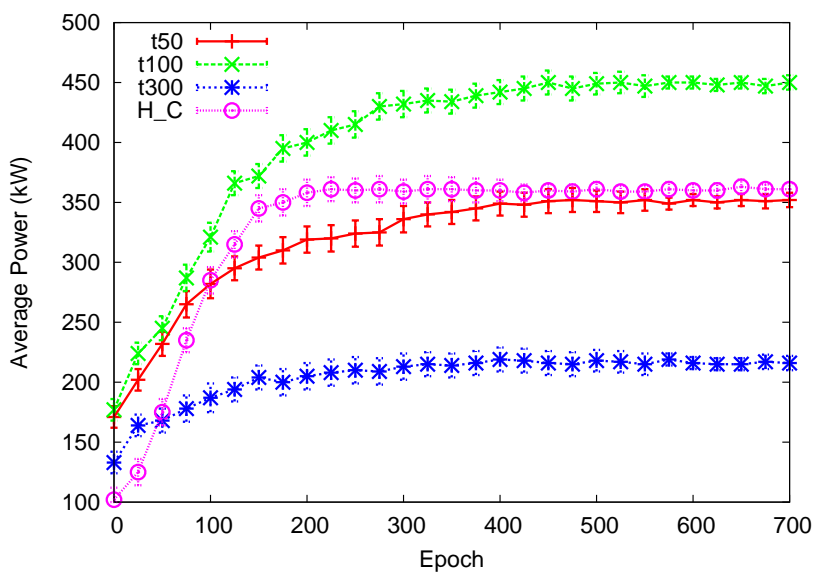

Figure 7: Comparison of evolution of ballast cuts while varying control loop times (50, 100, and 300 minutes) versus the ballast cuts produced from hillclimbing (hill-climbing had a control loop time of 100 minutes)

\begin{tabular}{|c|c|c|c|c|}
\hline \multicolumn{5}{|c|}{ Neural network and AQWA comparison } \\
\hline Experiment & $\begin{array}{c}\text { Population } \\
\text { Size }\end{array}$ & Generations & $\begin{array}{c}\text { NN } \\
(\mathrm{hrs})\end{array}$ & $\begin{array}{c}\text { AQWA } \\
(\mathrm{hrs})\end{array}$ \\
\hline 1 Pwr Calc & $\mathrm{n} / \mathrm{a}$ & $\mathrm{n} / \mathrm{a}$ & $\sim 1 \mathrm{e}-3$ & $\sim 4$ \\
\hline EA & 100 & 1000 & $\sim 4$ & $\sim 400 \mathrm{e} 3$ \\
\hline EA & 500 & 1000 & $\sim 6$ & $\sim 2 \mathrm{e} 6$ \\
\hline
\end{tabular}

Table 2: Computational times for AQWA simulator, neural network function approximation, and the evolutionary algorithm

hours to run. If an AQWA simulation was run every time that the neural network was called, the evolutionary algorithm would take upwards of 2 million hours to complete. As population size or number of epochs increases to any realistic amount for an evolutionary algorithm, AQWA ceases to be a viable method for finding power output.

\section{DISCUSSION}

We used an evolutionary algorithm to successfully design the ballast chamber geometry of a WEC, given a specific WEC geometry. The key to running this evolutionary algorithm was the neural network function approximator, which drastically reduced the computational time of calculating power outputs. The key difference between our work and traditional WEC design processes is the implementation of this function approximator. By reducing the time it takes to analyze one specific design, we were able to consider many more candidate designs than in a normal WEC design process.

Another important difference between our research and the traditional WEC design process is the implementation of the evolutionary algorithm. WEC design generally involves a human expert, who often imparts bias into the analysis (intentionally or unintentionally). By automating the design process with an evolutionary algorithm, the need for in-depth domain knowledge is eliminated, because more de- 
signs may be considered and the designs are automatically searched through in an intelligent manner.

The algorithm presented in this paper could potentially be extended to many other problems, especially similar problems in wave energy. We optimized the ballast geometry for a specific WEC geometry, but there is no apparent reason why this algorithm couldn't be extended to other geometries. For any arbitrary WEC geometry, if a function approximation for power can be developed using AQWA simulation data, then that function approximation could be used in a time domain simulation similar to the one we used, to predict annual power of the WEC as a function of chosen design parameters. More generally, any problem which could be solved with a population based search but requires time-consuming simulations could potentially be solved using an algorithm similar to the one presented in this paper. Future research of this algorithm in other domains could potentially demonstrate that substituting a simulation with a faster function approximation could result in a greatly improved design process.

\section{Acknowledgements}

We would like to thank everyone at Columbia Power, especially Ken Rhinefrank and Al Schacher for all their invaluable help, without which this project could not have been completed. We would also like to thank Zhe Zhang, for his help with using the AQWA software package.

This work was partially supported by Columbia Power under grant 2010-1906.

\section{REFERENCES}

[1] Ansys AQWA: Elucidative programming, 2010.

[2] A. Agogino and K. Tumer. Efficient evaluation functions for evolving coordination. Evolutionary Computation, 2008.

[3] T. Chen and R. Chen. Universal approximation to nonlinear operators by neural networks with arbitrary activation functions and its application to dynamical systems, 1995.

[4] K. Deb. Evolutionary algorithms for multi-criterion optimization in engineering design, 1999.

[5] F. Farley and R. Rainey. Radical design options for wave-profiling wave energy converters. 21st International Workshop on Water Waves and Floating Bodies, April 2006.
[6] A. Globus, G. Hornby, D. Linden, and J. Lohn. Automated antenna design with evolutionary algorithms. AAIA Space 2006 Conference, 2006.

[7] G. S. Hornby, S. Takamura, T. Yamamoto, and M. Fujita. Autonomous evolution of dynamic gaits with two quadruped robots. IEEE Transactions on Robotics, 21:402-410, 2005.

[8] J. Hudson, M. Kube, R. Adomaitis, I. Kevrekidis, A. Lapedes, and R. Farbar. Nonlinear signal processing using neural networks: Prediction and system modeling. In Los Alamos National Laboratory Theoretical Division, pages 2075-2081, July 1987.

[9] R. Leupers and F. David. A uniform optimization technique for offset assignment problems. In Code Optimization Techniques for Embedded Processors: Methods, Algorithms, and Tools. 2000.

[10] P. X. Liu, M. J. Zuo, and M. Q.-H. Meng. Using neural network function approximation for optimal design of continuous-state parallel-series systems. Computers $\&$ Operations research, 30(339-352), July 2001.

[11] M. McCormick. Ocean Wave Energy Conversion. John Wiley and Sons, New York, 1981.

[12] D. Mollison, O. Buneman, and S. Salter. Wave power availability in the ne atlantic. Letters to Nature, 1976.

[13] M. Mueller. Electrical generators for direct drive wave energy converters. In IEE Proceedings. IET, February 2002.

[14] K. Narendra and K. Parthasarathy. Identification and control of dynamical systems using neural networks. IEEE Transactions on Neural Networks, March 1990.

[15] D. Ross. Power from the Waves. Oxford University Press, New York, 1995.

[16] W. Sarle. Neural networks and statistical models. In Proceedings of the Nineteenth Annual SAS Users Group International Conference, pages 84-89, April 1994.

[17] E. J. Shaughnessy, I. M. Katz, and J. P. Schaffer. Introduction to Fluid Mechanics. Oxford University Press, New York, 2005.

[18] J. Wieczorek, O. Gol, and Z. Michalewicz. An evolutionary algorithm for the optimal design of induction motors. IEEE Transactions on Magnetics, November 1998. 